\title{
ATE AND AIRCRAFT MECHANICAL DIAGNOSTICS
}

\author{
U.S. Army Aviation Research and Development Command \\ St. Louis, Missouri 63166
}

\section{ABSTRACT}

The Army is committed to fielding a family of automatic test support systems (ATSS) to support all Army materiel. The current plan is to field the AN/USM-410 "third generation" ATE. The Army has also sponsored a significant amount of work ained at achieving an "on condition" maintenance philosophy for major aircraft subsystems by monitoring turbine engine performance, engine mechanical condition, health of various rotating dynamic components, hydraulic systems, and structural integrity of the aircraft. The objective is to investigate the viability of accomplishing the aircraft condition monitoring and diagnostic task using ATE. Significant points are the following: non-intrusive monitoring of aircraft mechanical systems is possible; impressive benefits can be attributed to this approach, but several attempts at fielding such a system have been terminated because of excessive initial investment costs; and mechanical diagnostics is not a simple extension of a measurement capability.

\section{INTRODUCTION}

The complexity and expense of maintaining modern electronic systems has made the investment in sophisticated Automatic Test Equipment (ATE) economically attractive. Similarly, aircraft mechanical systems are subject to the same trends. At this point, two definitions are in order. First, the term "Aircraft Mechanical Diagnostics" generally applies to techniques of the condition monitoring, fault isolation and trend monitoring or prognosis of the aircraft powerplant, the drive train, and various functional and structural components. Many degrees of monitoring, techniques, and system configurations are available and will be discussed later. The term "ATE" generally refers to all types of electronic test systems, monitoring, or diagnostic hardware that operates either automatically or semi-automatically. Although there are potentially many variations of ATE available which could easily be interfaced with an aircraft mechanical diagnostic system, in the interest of simplicity and reality, hereafter when referring to ATE we will be referring to a generic third generation ATE. The main reason for restricting ourselves to the third generation ATE is that the Army is committed to fielding a family of Automatic Test Support Systems (ATSS). The first step is to field an interim system, the AN/USM 10 which is a third generation system.

Briefly, the third generation ATE involves the generation and measurement of analog waveforms and digital data by using data sampling and digital conversion techniques under software control. No attempt will be made to discuss the impact of distributed microprocessors or fourth generation ATE on this subject.

The obvious need for knowing the aircraft condition is that in-flight system failures have the potential of producing disasterous results. This need is not a major requirement for automated diagnostics because the manual performance monitoring techniques, the physical inspections and the mandatory time change of components provides a high margin of safety for aircraft. This then alludes to the primary need for sophisticated and comprehensive automated diagnostics. Aircraft maintenance is expensive. This high cost is attributable to the fact that physical inspections take time in both maintenance manhours and aircraft downtime, quite of ten require disassembly of components thus introducing the possibility of causing problems, and, moreover, can be somewhat subjective. Mandatory time changes increase the logistics requirements and also require excessive maintenance manhours and aircraft downtine. Concisely stated, the automation of condition monitoring and diagnostics provides improvements in flight safety through early fault detection; allows better utilization of skilled personnel by reducing routine inspection and trouble-shooting tasks; allows major cost savings attributable to reductions in unwarranted removals, excessive spare parts consumption, and secondary damage; and facilitates an "ON CONDITION" maintenance philosophy resulting in extension or elimination of mandatory time changes. Another factor to consider is that some unscheduled maintenance is performed on the basis of flight crew squawks. In this case automated in-flight data can easily verify and isolate the source of the squawk, thus saving the time and effort of reproducing the same conditions.

\section{AIRCRAFT MECHANICAL DIAGNOSTICS}

Many configurations of aircraft diagnostic systems with varied capabilities have been tested. They range from the complex and comprehenstve systems such as the Army's Automatic Inspection, Diagnostic and Prognostic System (AIDAPS) 1 and 


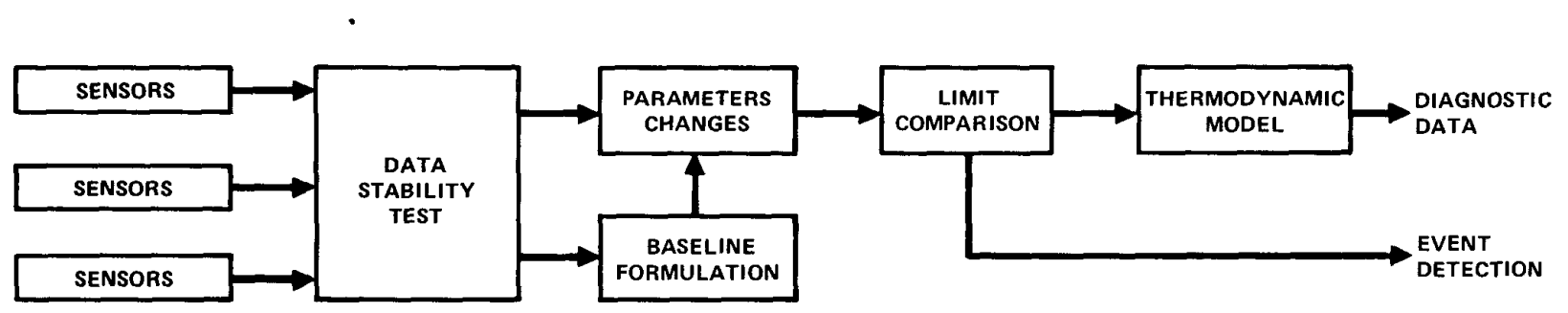

\section{PARAMETERS MEASUREMENTS \\ - temperatures \\ - pRessures \\ - ShAFT SPEeds \\ - OUTPUT HORSEPOWER \\ - fuel flow}

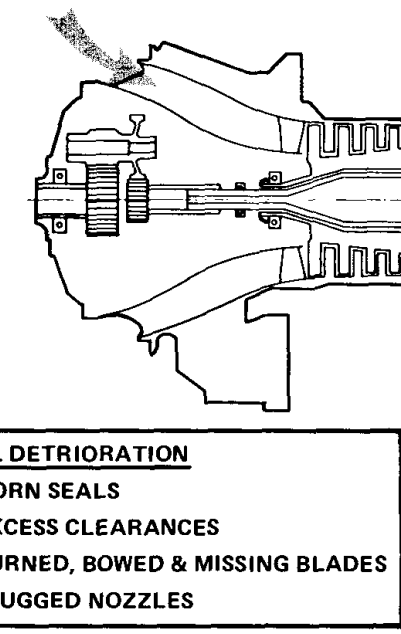

FIGURE 1 ENGINE PERFORMANCE MODEL

the Navy's In-flight Englne Condition Monitoring System (IECMS) ${ }^{2}$, both of which automatically monitor and fault isolate to a line replaceable unit to the manual methods of trending engine performance ${ }_{3}$ as implemented by Army with its TEACHIT program and the AF SAC with its ${ }_{4}$ Diagnostic Guide - Engine Condition Monitoring. There are also various single parameter monitors such as in line oil analyzers, various types of chip detectors, and temperature history recorders. The diagnostic systems all share certain general characteristics: a sensing device, a signal conditioning function, a processing or decision making function, and some form of display. In many instances these functions involve a considerable electronic capability.

There are three significant functional areas: engine performance, mechanical systems, and structural integrity. Engine performance monitoring can be performed at various levels of sophistication and cost ranging from a single point check of turbine temperature which is trended with time through a complete thermodynamic analysis of engine performance. The single point measurement has generally been taken manually using cockpit instrumentation at a given operating condition, corrected to standard conditions, and trended against time. From this, gross deviations in engine performance can be detected. However, this technique is subject to both human errors and instrumentation errors because there is no cross check of the data. Even with these faults, extensive savings have been documented. The next level of sophistication involves

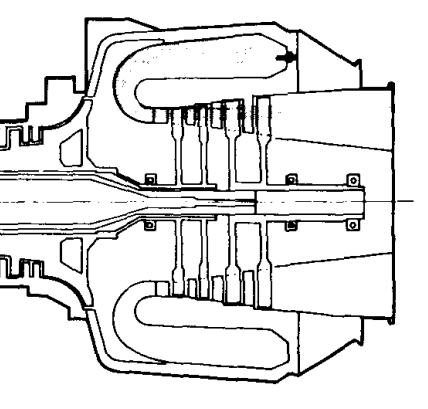

PERFORMANCE MEASUREMENTS

- efFiciencies

- pumping capacitr

- nozzle areas

- temperature profile

- flow leakage

\begin{tabular}{|lc|}
\hline \multicolumn{2}{c|}{ ISOLATION } \\
\cline { 2 - 2 } - COMPRESSOR & $\bullet$ TURBINE INLET \\
- GAS PRODUCER & TEMPERATURE \\
TURBINE & $\bullet$ COMBUSTOR (FUEL \\
- POWER TURBINE & NOZZLES) \\
- 1ST STAGE GP & INLET GUIDE \\
TURBINE NOZZLE & VANE CONTROL \\
& $\bullet$ SENSORS/SYSTEM \\
\hline
\end{tabular}

automatically recording several data points at a given operating condition, smoothing the data thus eliminating transient readings, and automatically applying corrections to standard conditions. In addition to the higher quality data obtained, data can be gathered at any stage of flight regardless of crew workload and physical constraints. This makes it possible to capture short term excursions of data or limit exceedences. However, this approach still suffers from some deficiencies. First, many failure modes are self compensating and thus one or two parameters are not sufficient to detect the incipient failure. Secondly, manual interpretation of results is still required. Thirdly, the parameters which can be monitored directly are not the best indicators of engine condition.

The latest generation of turbine engine health monitoring techniques involves a complete or nearly complete thermodynamic analysis of the engine or a gas path analysis. Faults in the gas path portion of the engine are detected by comparing previously recorded data from the baseline (or known good condition) with actual engine performance. The most efficient indicators are the fundamental performance characteristics of the engine such as efficiencies, nozzle areas, and pumping capacities. These performance characteristics are calculated from the measureable engine parameters such as temperatures, pressures, speeds, etc. The governing relationships are derived from a set of differential equations which define the thermodynamic model of the engine. Figure 1 contains the measured engine parameters and the engine 
performance characteristics for a relativeiy simple engine: the Lycoming T-53-L13. Figure 2 is a flow chart for the $\mathrm{T}-53$ engine logic.

Fault condition limits can be set that indicate when the deterioration has reached the point at which maintenance action is required. The deterioration can then be isolated to a particular module of the engine by further pattern recognition analysts of the measured and calculated parameters. The gas path fault detection logic implemented for the $\mathrm{T}-53$ engine included additional features of sensor diagnostics and detection confidence level calculations that Indicated whether or not a detected fault indication was likely to be due to sensor failure (e.g., drift error or total failure) rather than a performance-related engine fault. These features improved the accuracy of the engine condition diagnosis.

The mechanical systems can generally be segregated into two categories, the monitoring of the rotating dynamic components such as the engine accessories and in the case of turboshaft engines the drive train and the monitoring of the various aircraft functional items such as pressures, temperatures, speeds, ect. Many of these latter tests require nothing more than monitoring a discrete signal. The former task of monitoring the rotating dynamic components, mainly gears and bearings, is more technically challenging. The two most practical methods are vibration analysis and oil debris analysis. The theory of debris analysis is quite simple. As a component deteriorates, metallic debris is generated. The condition of the component is then a function of the amount, rate, and type of debris generation. A number of devices for measuring this are available, including magnetic chip detectors, indicating screens, turbidity monitors. One problem is that there has been difficulty in separating normal wear indications from catastrophic failure indications. Major efforts are underway to solve this problem. Thus, oil debris analysis can be considered a viable technique for testing equipment with circulating oil systems. However, this technique will not work for grease lubricated systems nor for systems with small oil reservoirs.

The other approach, vibration analysis, is more adaptable but more complex. The large computational capability required has previously made

FIGURE 2 THERMODYNAMIC ANALYSIS LOGIC DIAGRAM

\section{MEASUREMENTS}
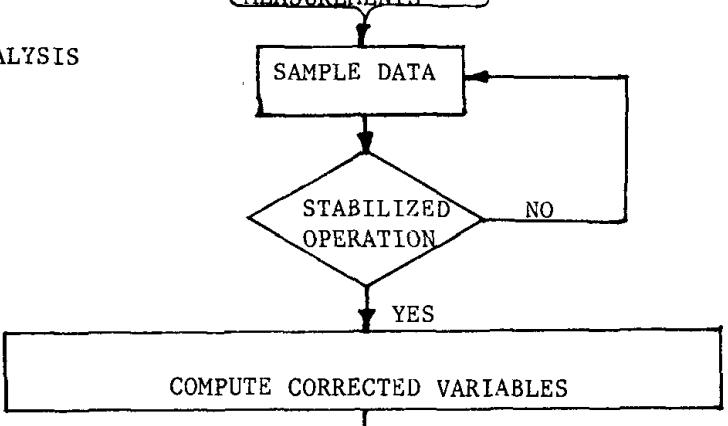

COMPUTE CORRECTED VARIABLES

COMPUTE VARIATIONS OF CORRECTEO

VARIABLES FROM THEIR BASELINES
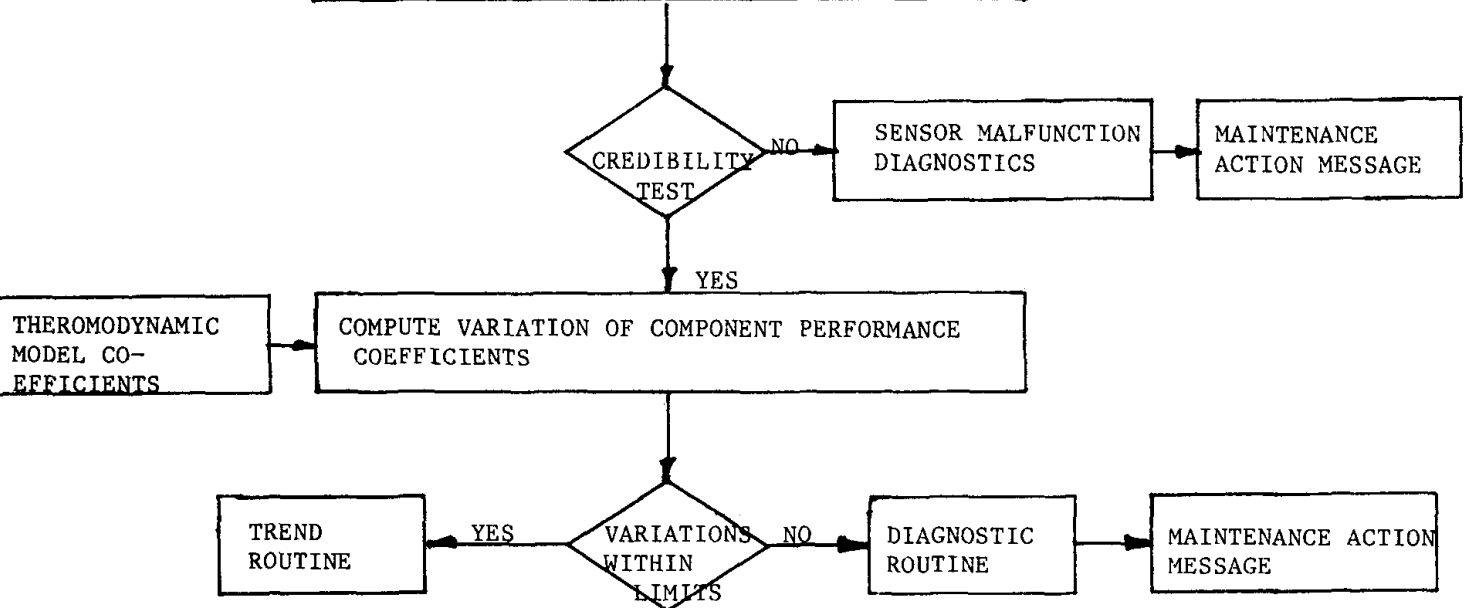
FIGUKE S COMPARISON OF VIBRATIUN ANALYSYS TELHNLUUED

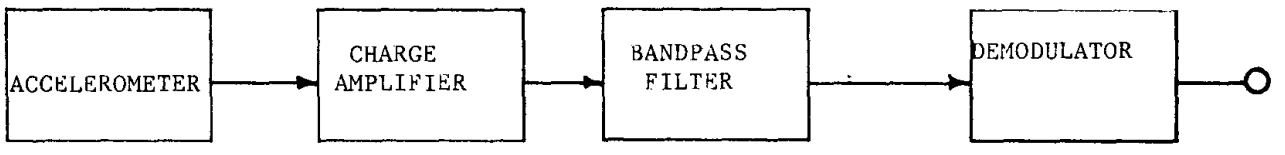

SHOCK PULSE

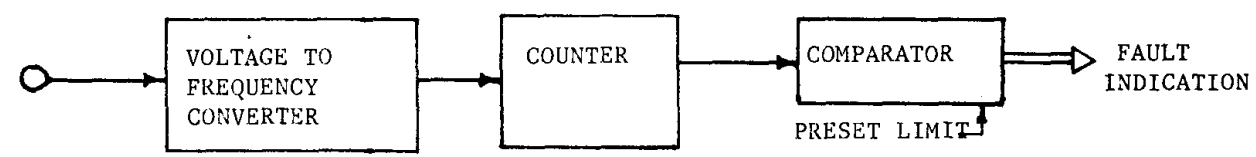

HFRT

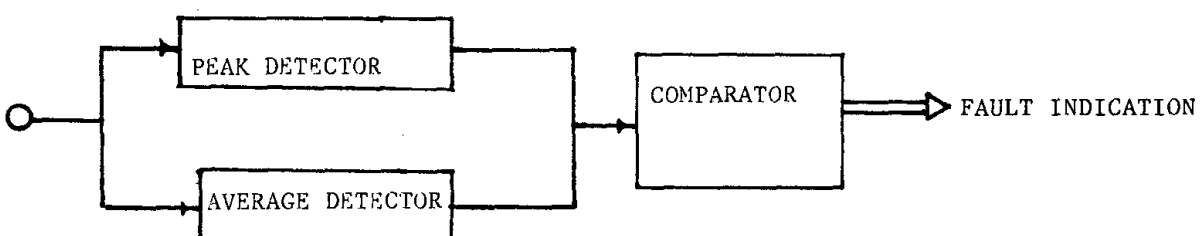

IFD

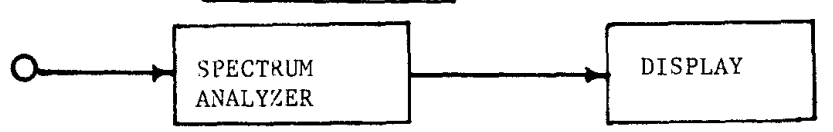

this approach undesirable. Recent advances in mechanical monitoring techniques and electronics are alleviating this problem.

Many approaches to vibration monitoring have been taken with varied degrees of success. The first approach was to monitor the overall vibration level. This proved to be inadequate, except for the highest level of defects. The next approach taken was pattern recognition within the range of 0 to $40,000 \mathrm{~Hz}$. The problem here is that the large data base required to develop the baseline characteristics and the large signal processing capability needed to accurately discriminate the various conditons results in a system which is complex and expensive. In spite of these problems the pattern recognition approach has been shown to be fairly effective and may in certain applications be the preferred technique. However, for most applications newer techniques would be preferred.

These later techniques such as Shock Pulse Analysis, Incipient Failure Detection $\left(\right.$ IFD) ${ }^{8}$ and the High Frequency Resonancy Techniques all have been shown to be more effective and less costly to implement than pattern recognition techniques. All have been shown to operate with generic baselines rather than custom baselines. Essentially, these systems are quite similar in that they all operate above $20,000 \mathrm{~Hz}$ and all use demodulated signals which have been band-pass filtered in that spectrum. At that point these techniques diverge and either the signal to noise ratio, the RMS value or simple pattern recognition techniques are used as discriminants. Figure 3 illustrates the differencies schematically. These high frequency techniques have achieved accuracies of up to $99 \%$ with relatively low false alarm rates.

Structural monitoring is another area where the statistically based TBO along with physical inspections are dominant. The most common methods of monitoring the structure electronically are strain gages, acoustic emission, and the use of flight loads recorders. While strain gages will monitor the actual stress, they are not considered viable for field use because they are fragile, require calibration, and require sensitive analog electronics and a large number of gages to effectively monitor the total aircraft. Acoustic emission is certainly a vigble approach but much development work remains. The most viable approach is the flight loads recorder. Here the aircraft maneuvers are recorded, rated according to severity, tabulated, and compared to an allowable amount of stress which was determined in a flight test phase.

In summary, because of the advances in mechanical monitoring techniques, the increased knowledge of the mechanisms of failure and failure progression, the advances in diagnostic logic, and 


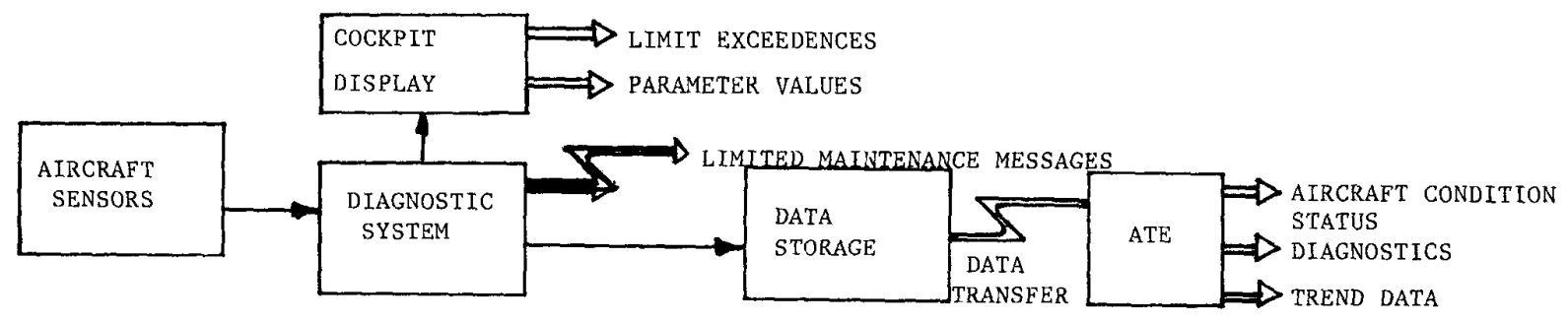

FIGURE 4 SYSTEM DIAGRAM

the test results of the referenced programs, it can be concluded that automated mechanical system condition monitoring and diagnosis is technically feasible and highly desirable.

\section{MECHANICAL DIAGNOSTICS AND ATE}

The need and electronic nature of mechanical diagnostic systems has been discussed as well as the fact that the affordability issue has been the demise of many systems. Also, the increased use of ATE in the field has been discussed. Thus, it seems reasonable that the integration of mechanical diagnostics into the ATE system should be investigated. The interface between the aircraft mechanical diagnostic system and the electronic support systems must be handled carefully. On the surface it would appear to be nothing more than a simple extension of a measurement capability. However, a review of the method of test will show several discrepancies. Once a radio or fire control black box is suspected of being faulty it can be removed from the aircraft and taken to the maintenance shop. There, with sophisticated test equipment, appropriate stimulus can be applied to the black box, the outputs measured, and judgements (either manual or automatic) as to the condition of the black box can be made. Appropriate corrective action can then be taken and the black box returned to service.

In the case of the aircraft mechanical systems it is generally impractical to remove them from the aircraft because of the labor involved. In most instances it is ever less practical to test thein away fron the aircraft because of operational requirements such as power, special fixtures, hydraulics, etc. In the case of the engine and transmission it is impossible because of the high power settings which are required. In sone instances it may even be necessary to be airborne. This is particularly true when the problem is a vibration from an unknown source, occurring intermittently. For example, much of the engine and transmission testing during the AIDAPS Program was accomplished in the air. Attempts at testing on the ground (tie-down node) resulted in unusual vibrations and damage to some of the components. This was on a UH-1H. No other aircraft were tested this way so there is no indication of what other problems may be encountered. A possible explanation of this phenomena is that flight loads are different from ground loads and it is not surprising that the drive train reacts differently. The conclusion here is that monitoring must be accomplished in service to be totally effective and that the data must be recorded and then transferred to the ATE Station for analysis.

\section{SYSTEM DEFINITION}

There are as many variations of system configurations as there are system designers. Since this is a general system designed without the aid of a detailed trade study, the significance of any particular paraneter or the segregation of any particular function is somewhat arbitrary.

Additional factors influencing the design must be considered. First, and most significant, is the employment concept of both the aircratt and the ATE. If the aircraft are deployed to diverse locations or if the avionics support is remote, then an off-board system is impractical. Hence, we will assume that an ATE system is to be used to support the avionics and that it is reasonably available both physically and functionally. Finally the proposed system must pass the affordability test. The system acquisition costs must be rasonable even though a higher cost system may be mire cost effective.

A minimum diagnostic system ${ }^{11}$ would consist of a general engine health check, drive train monitoring, operational limit monitoring, maintenance data recordings, and engine performance trending. By using this system as a core, adding a significant on-board data storage capability, and using the processing capability of the ATE, a more complete diagrostic system would be available. The on-board memory could be an optional feature but this would reduce the utility of the system. The system can best be described by the block diagram in Figure 4 .

The cockpit display would be used to display parameters upon request and alert the pilot to gross limit exceedances. All other data processing, health determinations, and diagnostics would be accomplished using the ATE. This system could be installed on a light helicopter for an estimated $\$ 10,000$. This is possible because of the advances in electronics and the reduced cost of sensors. 'the use of a data bus would also 
reduce the cost and enhance the system performance. More complex requirements than described here have been accomplished with 8000 twenty bit words of memory in the airborne portion and 32,000 words on the ground.

The point here is that the electronic capability is well within todays technology.

\section{BENEFIT ANALYSIS}

Several benefit analyses have been accomplished on diagnostic systems. On a simple diagnostic system in a commercial environment it was estimated that the savings would equal the cost in about a year. However, in the military environment where aircraft usage is significantly below 100 hours/ months, it would take 2 to 3 years to recover the costs. The sources of these savings were reduced overall costs and a reduction in accident damage due to mechanical failures. Not considered, but certainly contributing to savings, would be reduced inspections, extended operating limits and reduced trouble-shooting times.

In the military environment with more sophisticated helicopters it was estimated that systems with similar capabilities would also be cost effective. By considering only the savings in maintenance costs, it was estimated that the benefit to cost ratio would be 2 to I for a UH-1H and 10 to 1 for a new helicopter. ${ }^{12}$ The conclusion is that this type of diagnostic systems is cost effective and that with lower initial investment costs because of the use of the ATE could be even more cost effective. The reasons that the new helicopter application is more cost effective is that the installation costs are lower during production as opposed to retrofit costs and that testability is considered during the mechanical system design.

\section{POTENTIAL APPLICATIONS}

Once committed to a system it is reasonable to investigate additional applications or expansions of functions. This is particularly true of diagnostic systems. Since the diagnostic system by definition monitors the performance of the aircraft, the most obvious addition is a crash recording capability. The impact would be the cost of hardening of the data storage media against shock and heat. The minimal inputs are drive train health, engine performance and aircraft position. With the modern mas memory capabilities and improved voice compreassion techniques even a voice channel could be recorded.

Another capability which is easily added is power available indicator. If the configuration selected has on-board engine performance capability and health monitoring, it is then possible to calculate the max. power available under the existing conditions and state of health of the engine. There is only then the problem of displaying the information to the pilot.
The most efficient use of this electronic capability would be to include the cockpit condition monitoring instruments in the system. A digital display certainly would take up less instrument panel space than current instrumentation. If carried to its maximum potential only the health status would be displayed rather than the parameter values.

\section{CONCLUSION}

It has been shown that it is technically feasible and cost effective for an aircraft mechanical diagnostic system to operate as part of the ATE. The importance of testability in early design has a large impact on cost effectiveness. The other major factor influencing cost effectiveness is the employment concept for the aircraft and the ATE. REFERENCES

I. Loretz, N.F., "Application of Gas Turbine Engine Condition Monitoring System to On-Condition Maintenance Programs." Paper No. 76-13476 ASME Gas Turbine Conf, Oct 1976.

2. Downing, N.L., "Using In-flight Engine Condition Monitoring System to Improve TF 41 Engine Survivability" Report JTCG/AS-74 T-010 Sept 1976.

3. McHugh, CW2 Peter C., "TEAC/HIT" US Army Aviation Digest Oct 1973.

4. Pettigrew, LTC James, L; McCord CM SGT Robert $M$, "Diagnostic Guide-Engine Condition Monitoring", Feb 1977.

5. Pettigrew, LTC James L, McCord, CM SGI Robert M., "Engine In-F1ight Monitoring Update," Combat Crew, July 1977.

6. "AIDAPS Program - Fifth Interin Technical Report Addendum 1," Garrett AiResearch Report No 76-12372; Aug 1976.

7. Mayer, T.C. Covill, G. F., George, J.A. Harrington, J.T., "Applications of the Shock Pulse Techniques to Helicopter Diagnostics," USAAVSCOM TR 77-21, Feb 75.

8. Board, David B., "Incipient Failure Detection in High Speed Rotating Machinery" 10th Symposium on Non-Destructive Evaluation, Apr 75.

9. Hickey, J.V., "AIMAPS, Test Cell Data Collection, USAAVRADCOM TR 78-4, Aug 1977.

10. Bailey, C.D., Pless, W.M. "Acoustic EmissionMonitoring Fatigue Cracks in Aircraft Structure" 22nd International Instrumentation; May 25-27 1976.

11. Murphy, J.A., "Diagnostic System Requirements for Helicopter Propulsion Systems" Journal of Aircraft VoI 15 No. 6, June 1978.

12. "AIDAPS Program - Final Technical Report" USAAVRADCOM TR-78-5, Oct 1977. 